\title{
Methods for Measurement and Verification of Purity of Iodine Cells for Laser Frequency Stabilization
}

\author{
J. Hrabina, P. Jedlička, and J. Lazar \\ Institute of Scientific Instruments, Academy of Sciences of the Czech Republic, \\ Královopolská 147, 61264 Brno, Czech Republic, shane@isibrno.cz
}

\begin{abstract}
We present an improved technique for detection of trace impurities in iodine-filled absorption cells for laser frequency stabilization. The results of purity investigation are compared to frequency shifts measured with a set of two iodine stabilized Nd:YAG lasers. The setup for direct fluorescence measurement with an Argon-ion laser operating at $502 \mathbf{~ n m}$ wavelength is equipped with compensation for laser power and spectral instabilities.
\end{abstract}

Keywords: stabilized laser, saturated absorption, spectroscopy, iodine

\section{INTRODUCTION}

$\mathrm{O}^{\prime}$ PTICAL FREQUENCY synthesis in the field of laser metrology is becoming mainstream in these days due to the great advances in femtosecond laser technology. Frequency locking of such comb generators may be done in the radiofrequency domain to atomic clocks extending their stability into the optical spectral range or in the reverse direction to high stability lasers and also to generate rf optical clocks. Nd:YAG stabilized lasers seem to be a suitable option thanks to the relative simplicity of the stabilization scheme and stabilities that can be achieved are not far from those of the rf cesium clocks.

Stabilization on the basis of saturated absorption in molecular iodine may be called a traditional method and due to good signal-to-noise ratio at the $532 \mathrm{~nm}$ of frequency doubled Nd:YAG laser gives very good results. Good relative stability can be easily achieved with a low-noise laser source and properly designed stabilization scheme and control electronics but the absolute value of the optical frequency is given by center frequencies of hyperfine components of the iodine transitions. Only near-ideal purity of the iodine in the absorption cell can result in optical frequencies corresponding to theoretical values.

We present a technology of iodine cell preparation that is a result of a long time development and verification by an independent method based on measurement of induced fluorescence and evaluation by the Stern-Volmer formula which has been used by the Bureau International des Poids et Mesures (BIPM) so there is a chance to compare our results [1], [2], [3]. The level of induced fluorescence intensity here is limited by several relaxation processes such as ionization, predissociation and collisional quenching. The quenching nonradiative transitions - can be caused by collisions with either iodine molecules or by collisions with molecules or atoms of impurities (foreign-gas quenching). The last mentioned process is the one that reflects the purity by reducing the lifetime of a state and can be evaluated by monitoring the spontaneous emission level from the irradiated cell. The main goal of this effort is to recognize the limits of the absolute precision of the optical frequency of iodine transitions and look for further improvements in the iodine cell manufacturing technology that may lead to even smaller frequency shifts.
This technique was introduced into the metrology practice with relation to $\mathrm{He}-\mathrm{Ne}$ iodine stabilized lasers. With the stability that can be reached with iodine stabilized frequencydoubled Nd:YAG lasers the chance to compare the purity investigation with measured frequency shifts could go even below the $\mathrm{kHz}$ level [4], [5], [6].

\section{MEASUREMENT OF INDUCED FLUORESCENCE}

Our experimental arrangement is based on measurement of the level of fluorescence in the irradiated iodine cell with an Argon-ion laser for excitation of iodine. The most suitable seems the $502 \mathrm{~nm}$ wavelength coinciding with absorption lines in iodine characterized by a high sensitivity to collisional quenching that is caused by a long lifetime of the excited level [4]. The strongest absorption line within the spectral width of the laser is the $\mathrm{R}(26) 62-0$ of the ${ }^{127} \mathrm{I}_{2}$ which contributes predominantly to the fluorescence measured.

The laser operates mostly in a multimode regime, so the reproducibility of the measured data was strongly influenced by variations of the spectral component of the laser output power coinciding with the absorption profile of the $R(26) 62-0$ line. Beside the overall power monitoring we decided to add one more level of compensation of the laser instability with the help of a reference iodine cell. The reference cell with an identical arrangement of a photomultiplier and optics is held at a constant cold finger temperature a little below the laboratory environment temperature. The output signal from the reference photomultiplier and reference detection chain with identical phase-sensitive detection relates to the fluorescence detected at the line of interest and thus to the laser power within the absorption line profile, Fig.1.

The presence of a stray light arising from the iodine cell proved to be very difficult to suppress completely. This problem depended strongly on the configuration of the iodine cell itself and was small in case of longer and large diameter cells. To separate the value of the stray light level from the total signal, we used cooling of the iodine cell cold finger with liquid nitrogen. This reduces the pressure of the iodine vapor down to the negligible level where no fluorescence could be detected. At this moment the photomultiplier detects only the stray light. Later, during the measurement the level of 
background signal due to stray light varies as well but varies corresponding to the overall output power of the laser. The compensation of the effect of stray light is based on monitoring of the laser power by a photodetector. In our configuration we used the output from the photodetector also as a source of reference signal for phase-sensitive detection with lock-in amplifiers to avoid unwanted phase jitter caused by phase-lock loop control of the laser beam chopper.

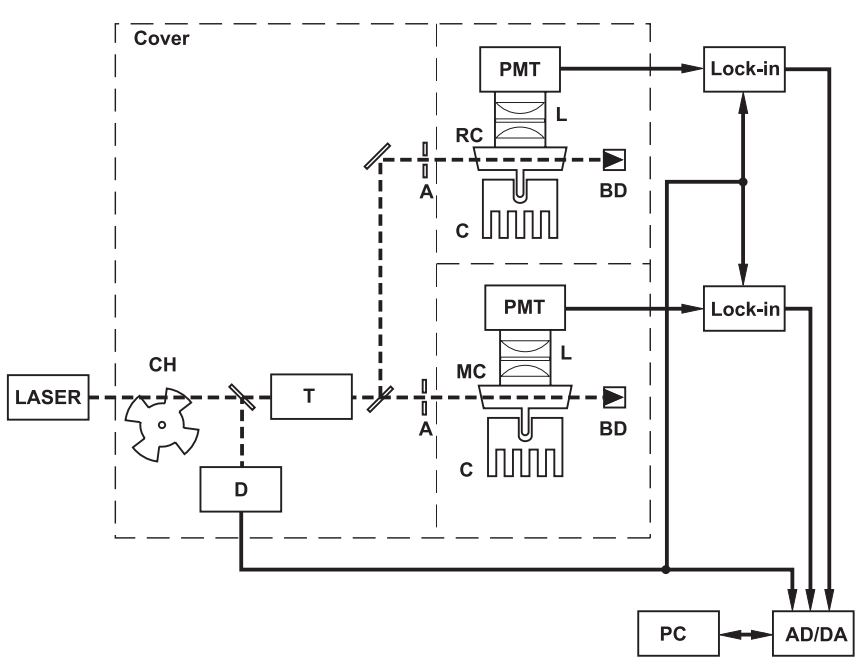

Fig.1 Experimental arrangement of the iodine excitation and fluorescence measuring apparatus. $\mathrm{CH}$ - chopper; $\mathrm{T}$ - telescope;

$\mathrm{D}$ - photodetector; A - aperture; PMT - photomultiplier; BD - beam dump; C - Peltier cooler with a radiator.

Laser source envelope linewidth of the laser running at maximum power is up to $5 \mathrm{GHz}$ while the Doppler-broadened absorption lines of iodine namely the $\mathrm{R}(26) 62-0$ do not exceed $0.6 \mathrm{GHz}$. Fluctuations of the spectral power density are a source of additional noise in the detection chain. They can be partially eliminated by a longer averaging time and filtering but there is also a long-time drift present which may influence the whole set of measurements lasting dozens of minutes. We added another set of fluorescence measurement with a reference cell and identical optical and photodetector setup, which monitors the amount of optical power corresponding to the absorption profile of our interest. The cell cold finger is kept at constant temperature and thus with constant pressure of gaseous iodine during the measurement process the detected intensity of fluorescence can be used to compensate the varying distribution of power spectral density.

The reference cell, as well as the measured cell, suffers from the effects of stray-light. To separate all these effects preparation of the measurement starts with cooling of both cold fingers with liquid nitrogen until all fluorescence fades away and the ratio of detected stray-light is recorded for the reference cell detection chain and for the measured cell as well, with respect to the power monitored by the photodetector. During the whole measurement process the normalized level of stray-light is subtracted in both detection channels. The level of detected fluorescence in the measuring channel is than compensated with respect to the reference one (Fig.2).

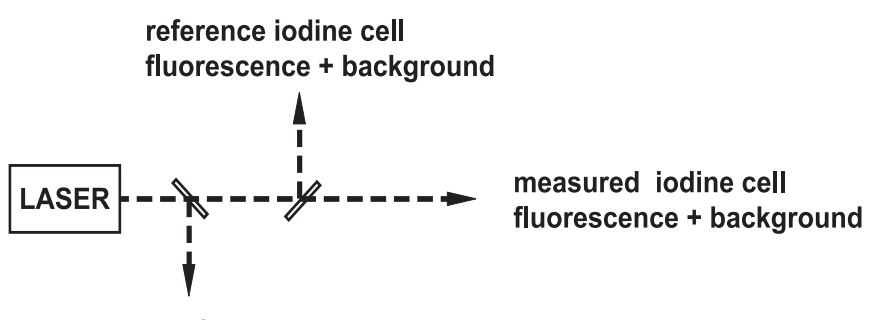

monitoring of the laser power $\approx$ background

Fig.2 Distribution of the laser output power for compensation of the frequency noise and stray light.

The value of the useful signal derived from the compensation through the reference cell becomes:

$$
I_{m e . c o r r}=I_{m e} \frac{I_{r e f .0}}{I_{r e f}}
$$

where $I_{m e}$ is the intensity of fluorescence detected with a photomultiplier on the measured cell, $I_{r e f}$ is the intensity of fluorescence detected on the reference cell at the same moment, $I_{\text {ref.o }}$ is the intensity of fluorescence detected on the reference cell at the beginning of the set of measurements and $I_{\text {me.corr }}$ is the corrected value of fluorescence on the measured cell.

The Stern-Volmer diagram shows a dependence of the reciprocal intensity of the detected fluorescence related to the intensity equivalent to $10 \mathrm{~Pa}$ iodine pressure on the inverted iodine pressure. It is based on the normalized Stern-Volmer formula that makes the measurement of the level of quenching possible and reproducible while the detection of the total value of fluorescence is technically impossible. The parameter from the normalized Stern-Volmer formula which can express the level of collisional quenching caused by impurities in the cell is the $\mathrm{K}_{0}$ coefficient representing the slope of the line of the diagram in log-log scale.

\section{IODINE CELL TECHNOLOGY}

Cells made at our institute are made of fused silica glass. This material allows perfect vacuum processing at a high temperature and thus additional releasing of gasses from the walls of the cell is eliminated. Joints between the cell tube and optical windows are a critical problem. We used either welding or soldering at high temperature over $1000^{\circ} \mathrm{C}$ with a special solder. This technology was preferred for the cells with Brewster angle windows. Iodine cells designed to operate in an extracavity arrangement for stabilization of frequency doubled Nd:YAG lasers are made with plane windows equipped with antireflection coatings on both sides of each window. The coatings are a traditional multilayer structure of $\mathrm{TiO}_{2}$ and $\mathrm{SiO}_{2}$, while the top covering layer is $\mathrm{SiO} 2$, the same material as the cell tube itself. This was intended to avoid any possible contamination of the cell.

Iodine cell filling process starts with distillation of the iodine from a commercial form into vacuum ampoules. The first distillation is done while the tubing is vacuum pumped. This first degree of distillation is a crucial one, when the iodine is freed from impurities adsorbed from its surface. The 
next distillation steps of the multistage process are performed only in vacuum between sealed ampoules with iodine and the impurities are removed by several types of molecular sieves. Finally the iodine is distilled into the evacuated and degassed cell via a break-seal and the cell is sealed. The iodine cells for $\mathrm{Nd}$ :YAG lasers we tested on impurities are $500 \mathrm{~mm}$ long to achieve acceptable signal-to-noise ratio even in a single-pass configuration. The cold finger is placed in the center and cell windows are antireflection coated, wedged and slightly tilted to further suppress reflections and etalon effects.

\section{MEASUREMENT OF FREQUENCY SHIFT}

We assembled a beat-frequency arrangement with two frequency doubled $532 \mathrm{~nm} \mathrm{Nd}$ :YAG lasers stabilized by the saturated absorption spectroscopy technique to detect frequency shifts caused by iodine cell impurities. The systems used were the iodine luminescent optical frequency standard ILP I2 /532-3L from Time Base, Düsseldorf, Germany with a prestabilization to a passive Fabry-Perot cavity through a frequency-modulation spectroscopy Pound-Drever technique and single Nd:YAG laser Prometheus from Innolight, Germany with the in-line configuration of the saturated absorption spectroscopy and third-harmonic detection chain and stabilization system of our design [7], [8]. The optical setup was designed to allow simple exchange of the iodine cells for frequency shift measurements.

Our first comparison was performed with a set of two cells tested to their purity by the evaluation of the Stern-Volmer coefficient through induced fluorescence measurement. Conditions of the stability recording and frequency shift measurement were kept at the levels mentioned in the recommendations issued by CIPM [9], [10].

To achieve good resolution of the beat signal to be measured by a counter (HP 53132A) we have chosen a pair of hyperfine components $\mathrm{a}_{10}$ and $\mathrm{a}_{11}$ of the $\mathrm{R}(56)$ 32-0 transition in molecular iodine ${ }^{127} \mathrm{I}_{2}$ that falls within a frequency range of $250 \mathrm{MHz}$ of the counter input which allows adjustments of comparator level and hysteresis. Both components are also close to the center of the Doppler-broadened R(56) 32-0 transition so it can be expected that the influence of the Doppler background slope to the frequency shift might be smaller. According to [10] the frequency difference is 126.513 $\mathrm{MHz}$ with an uncertainty of $1.5 \mathrm{kHz}$. Our estimation of the frequency shift is related to this value. Further improvement of signal-to-noise ratio of the beat signal was performed by a synchronous modulation of both laser systems which reduced interference of the modulation signals. Precise equalization of both amplitude and phase was adjusted by monitoring of the beat signal with a radiofrequency spectrum analyzer and reducing the signal linewidth down to the level of a beat signal of free running unmodulated lasers. Synchronization of the modulation signals was derived directly from a single quartz oscillator and following set of dividers generating the modulation signals with adjustable attenuator and phase shifter (Fig. 3). Result of the stability recording confirms the stabilities that can be achieved with frequency doubled $\mathrm{Nd}$ :YAG stabilized laser standards of optical frequencies and approaches the $10^{-14}$ level for integration time $100 \mathrm{~s}$. The recording of Allan variances in Fig. 4 shows that frequency noise of both systems is dominated by random noise fluctuations and the mean value of the detected frequency shift is not influenced by drifts. Together with careful elimination of DC offsets in the key components of the detection chain and servo loop we can consider the relative frequency shift of the pair of lasers predominantly to be the difference between absolute frequency shifts of hyperfine components of the iodine spectrum of the two cells.

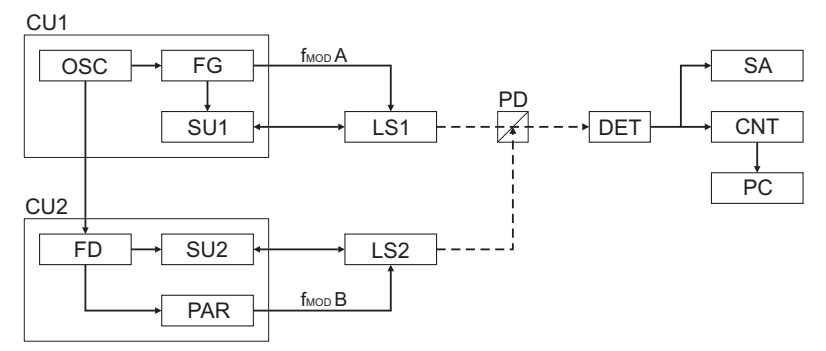

Fig.3 Simplified scheme of the arrangement for synchronization of amplitudes and phase of modulation signals of a pair of frequency doubled Nd:YAG iodine stabilized lasers and beat signal detection. CU1, CU2 - Control unit; OSC - Xtal oscillator;

FG - Fmod generator; SU1, SU2 - Stabilization unit;

FD - Frequency divider; PAR - phase and amplitude regulator; LS1, LS2 - Laser system; PD - polarization divider;

DET - photodetector; SA - spectral analyzer; CNT - counter; PC - computer.

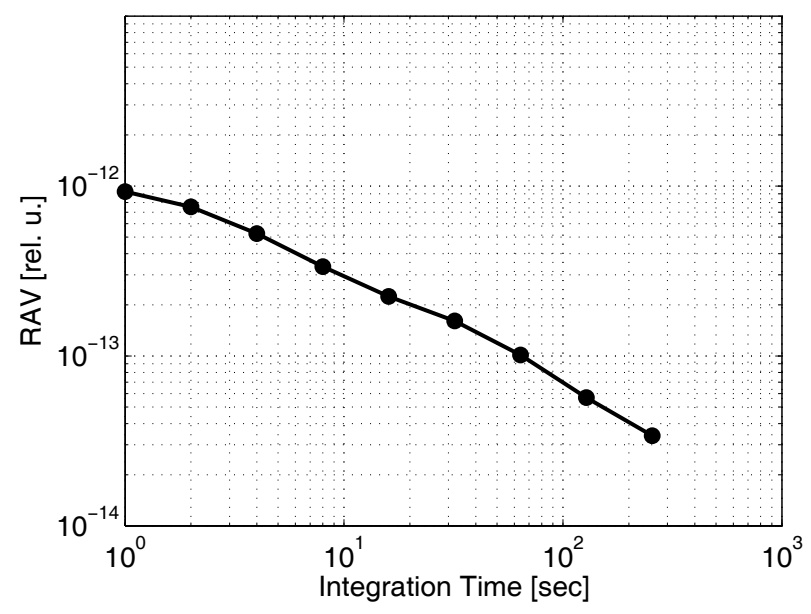

Fig.4 Recording of the Allan variances of the beat signal measurement between the two iodine stabilized frequency doubled Nd:YAG lasers.

We arranged the comparison on the basis of matrix measurement with two components where the estimated uncertainty is derived from the Allan variance record over 100 seconds of each measurement. The mean value from the frequency difference recordings shows that there are shifts present, most likely due to the difference in the slope of Doppler background in case of the two systems. We set the temperature of the cold finger of the ISI cell to the same temperature as the recommended $-10{ }^{\circ} \mathrm{C}$ of the Time Base system. The modulation signal for the third-derivative detection system of both lasers was kept at identical amplitude 
and phase to avoid not only problems with counting of the frequency-modulated beat signal but also to avoid frequency shifts caused by modulation width. Calculating the final frequency difference as a mean value from the two values of lasers locked to components $\mathrm{a}_{10}$, or $\mathrm{a}_{11}$ and vice versa seems to eliminate the Doppler background shifts (Tab.1).

Tab.1 Values of the frequency shift of the lasers locked to the $\mathrm{a}_{10}$ and $\mathrm{a}_{11}$ components of the $\mathrm{R}(56)$ 32-0 transition.

\begin{tabular}{|c|c|c|c|}
\cline { 3 - 4 } \multicolumn{2}{c|}{} & \multicolumn{2}{c|}{ Time Base system and cell } \\
\cline { 3 - 4 } \multicolumn{2}{c|}{} & $\mathrm{a}_{10}$ & $\mathrm{a}_{11}$ \\
\hline \multirow{3}{*}{$\begin{array}{c}\text { ISI system } \\
\text { and cell }\end{array}$} & $\mathrm{a}_{10}$ & & $\begin{array}{l}\Delta \mathrm{f}=3.3 \mathrm{kHz} \\
\sigma=5.10^{-14}\end{array}$ \\
\cline { 2 - 4 } & $\mathrm{a}_{11}$ & $\begin{array}{l}\Delta \mathrm{f}=-6.1 \mathrm{kHz} \\
\sigma=8.10^{-14}\end{array}$ & \\
\hline
\end{tabular}

\section{RESULTS AND CONCLUSION}

The final absolute value of laser frequency stabilized by means of subdoppler spectroscopy of iodine vapour is influenced by various other factors and effects, physical as well as technical but the quality (purity) of the cell is the most crucial one. Once the cell is sealed there is no way to change it and this technique of monitoring the impurity level in iodine cells seems to be the only chance how to distinguish the factors influencing the frequency precision. The other parameters with direct relation to the absolute laser frequency shift are of technical nature and can be measured and eliminated relatively easily, such as electronic offsets in the detection chain, iodine cell cold finger temperature, modulation amplitude, non-harmonic distortion of the modulation signal, etc.

Frequency doubled Nd:YAG lasers stabilized to saturation spectroscopy in iodine are able to reach or even overcome the $10^{-10}-10^{-14}$ limit of relative stability due to much better signalto-noise ratio achievable at the $532 \mathrm{~nm}$ wavelength compared to the traditional $\mathrm{He}-\mathrm{Ne}$ laser etalons operating at $633 \mathrm{~nm}$. This gives the chance to extend the precision of previous investigations of iodine cell purity and related frequency shifts performed with intracavity cells of $\mathrm{He}-\mathrm{Ne}$ lasers. Our first iodine cell comparison performed with two cells with SternVolmer coefficients $\mathrm{K}_{0}=0.85 \mathrm{~Pa}$ for the iodine cell of the Time Base system and $\mathrm{K}_{0}=1.7 \mathrm{~Pa}$ for the ISI system shows a frequency difference $\Delta \mathrm{f}=2.8 \mathrm{kHz}$ with a precision expressed by the Allan variances in Fig. 4 . The value $\mathrm{K}_{0}$ of the first cell is at the level extrapolated in [3] which is assumed to have a zero frequency shift. Relative frequency shift of $2.8 \mathrm{kHz}$ measured here fits into the relation observed here. It can be expected that with more iodine cells to come and more values of their frequency shifts, a more complex picture of the limits of not only relative stability but of the absolute precision that can be achieved by iodine stabilized Nd:YAG lasers below the $\mathrm{kHz}$ level will arise.

\section{ACKNOWLEDGEMENTS}

Authors wish to express thanks for support to the grant projects from Grant Agency of the Academy of Sciences of the Czech Republic, project no. A20065050, Ministry of Education, Youth and Sports of the Czech Republic, projects No.: LC06007, 2C06012, Academy of Science of the Czech Republic, projects No.: AV0 Z20650511 and KAN311610701, Ministry of Industry and Commerce, projects No: 2A1TP1/127, FT-TA3/133.

\section{REFERENCES}

[1] Stern, O., Volmer, M. (1919). Phys. Z. 20, 183-188.

[2] Spieweck, F. (1985). Influence of small impurities in absorption cells of $\mathrm{I}_{2}$ stabilized lasers upon their frequency. IEEE Trans. on Instrum. and Meas. IM-34, 246-248.

[3] Fredin-Picard, S. (1989). A study of Contamination in ${ }^{127} \mathrm{I}_{2} \quad$ Cells Using Laser-Induced Fluorescence. Metrologia 26, 235-244.

[4] Picard, S. et al. (2003). Comparison of ${ }^{127} \mathrm{I}_{2}$-stabilized frequency-doubled Nd:YAG lasers at the Bureau International des Poids et Mesures. Appl. Opt. 42, 10191028.

[5] Diddams, S.A. et al. (2000). Direct link between microwave and optical frequencies with a $300 \mathrm{THz}$ femtosecond laser comb. Phys. Rev. Lett. 84, 5102-5105.

[6] Nevsky, A. YU. et al. (2001). Frequency comparison and absolute frequency measurement of I2-stabilized lasers at 532nm. Optics Communications 192, 263-272.

[7] Holzwarth, R. et al. (2001), Absolute frequency measurement of iodine lines with a femtosecond optical synthesizer. Applied Physics B 73, 269-271.

[8] Drever, R.W.P., Hall, J.L., Kowalski, F.V., Hough, J., Ford, G.M., Munley, A.J., Ward, H. (1983). Laser phase and frequency stabilization using an optical-resonator. Appl. Phys. B 31, 97-105.

[9] Quinn, T.J. (1999). Practical realization of the metre. Metrologia 36, 211-244.

[10] BIPM (2003). Mise en pratique for the definition of the metre. Retrieved from http://www.bipm.fr/utils/common /pdf/mep/M-e-P_I2_532.pdf 